\title{
ERRATUM TO: MULTILEVEL DYNAMIC GENERALIZED STRUCTURED COMPONENT ANALYSIS FOR BRAIN CONNECTIVITY ANALYSIS IN FUNCTIONAL NEUROIMAGING DATA
}

KWANGHEE JUNG

THE UNIVERSITY OF TEXAS HEALTH SCIENCE CENTER AT HOUSTON

YOSHIO TAKANE

UNIVERSITY OF VICTORIA

HEUNGSUN HWANG

MCGILL UNIVERSITY

TODD S. WOODWARD

UNIVERSITY OF BRITISH COLUMBIA

\section{Erratum to: PSYCHOMETRIKA \\ DOI: 10.1007/s11336-015-9440-6}

\section{Correction 1:} and 6.

On page 9. There are two places to be corrected in the paragraph regarding Tables 5

"Table 5 shows that the variance estimate of each level-2 random loading $\left(\lambda_{j}{ }^{\prime} s\right)$ across the subjects and its standard error obtained from bootstrap samples. Here, the random loading denotes the deviation between the fixed loading and individual subject's estimate of the loading. Thus, the variance estimate of each level-2 random loading represents inter-subject variability in the loading. It was found that the variances of the random loadings for all observed variables turned out to be statistically significant. This suggests that there existed substantial subject-wise differences in each of the loadings. Table 6 exhibits the variance estimate of the Level-2 random path coefficient $\left(\theta_{i j}{ }^{\prime} s\right)$ of each latent variable and its standard error obtained from bootstrap samples. About 87\% of the variance estimates of the random path coefficients turned out to be statistically significant, suggesting substantial differences in each of the path coefficients across the subjects."

The parts indicated above should have read as follows:

"Table 5 shows that the variance estimate of each level-2 random loading $\left(\lambda_{j}{ }^{\prime} s\right)$ across the subjects and its standard error obtained from bootstrap samples. Here, the random loading denotes the deviation between the fixed loading and individual subject's estimate of the loading. Thus, the

The online version of the original article can be found under doi:10.1007/s11336-015-9440-6.

Correspondence should be made to Kwanghee Jung, Department of Pediatrics, Children's Learning Institute, The University of Texas Health Science Center at Houston, 7000 Fannin UCT 2373J, Houston, TX 77030, USA.

Email: kwanghee.jung@uth.tmc.edu 
variance estimate of each level-2 random loading represents inter-subject variability in the loading. It was found that the variances of the random loadings for about $71 \%$ of observed variables turned out to be statistically significant. This suggests that there existed substantial subject-wise differences in each of the loadings. Table 6 exhibits the variance estimate of the Level-2 random path coefficient $\left(\theta_{i j}{ }^{\prime} s\right)$ of each latent variable and its standard error obtained from bootstrap samples. About 91\% of the variance estimates of the random path coefficients turned out to be statistically significant, suggesting substantial differences in each of the path coefficients across the subjects."

Correction 2: The values of C.R. in Tables 5 and 6 should be as follows:

TABLE 5 .

The variance estimates of the Level-2 random loading estimates and their standard errors.

\begin{tabular}{|c|c|c|c|c|}
\hline \multicolumn{2}{|c|}{ The variance of $\lambda_{j}{ }^{\prime} s$} & \multirow{2}{*}{$\frac{\text { Estimate }}{0.001^{*}}$} & \multirow{2}{*}{$\frac{\text { S.E. }}{0.000}$} & \multirow{2}{*}{$\frac{\text { C.R. }}{2.92}$} \\
\hline IPL_R & 1 & & & \\
\hline & 2 & $0.002 *$ & 0.001 & 2.69 \\
\hline & 3 & $0.002 *$ & 0.001 & 2.00 \\
\hline & 4 & $0.001 *$ & 0.000 & 3.45 \\
\hline & 5 & $0.002 *$ & 0.001 & 2.43 \\
\hline \multirow[t]{5}{*}{ PreCG_R } & 1 & $0.008 *$ & 0.002 & 3.34 \\
\hline & 2 & $0.003^{*}$ & 0.001 & 3.56 \\
\hline & 3 & 0.018 & 0.016 & 1.09 \\
\hline & 4 & $0.003^{*}$ & 0.001 & 2.93 \\
\hline & 5 & $0.010^{*}$ & 0.003 & 3.44 \\
\hline \multirow[t]{5}{*}{ CL_R } & 1 & $0.001 *$ & 0.000 & 2.99 \\
\hline & 2 & 0.002 & 0.001 & 1.92 \\
\hline & 3 & $0.000 *$ & 0.000 & 2.23 \\
\hline & 4 & $0.001 *$ & 0.001 & 2.06 \\
\hline & 5 & 0.002 & 0.001 & 1.78 \\
\hline \multirow[t]{5}{*}{ CL_L } & 1 & 0.003 & 0.002 & 1.50 \\
\hline & 2 & 0.001 & 0.001 & 1.48 \\
\hline & 3 & $0.004 *$ & 0.001 & 3.32 \\
\hline & 4 & 0.001 & 0.001 & 1.76 \\
\hline & 5 & $0.001 *$ & 0.000 & 2.83 \\
\hline \multirow[t]{5}{*}{ IPL_L } & 1 & $0.003 *$ & 0.001 & 2.80 \\
\hline & 2 & 0.004 & 0.003 & 1.61 \\
\hline & 3 & $0.001 *$ & 0.000 & 3.18 \\
\hline & 4 & $0.001 *$ & 0.000 & 3.12 \\
\hline & 5 & $0.004 *$ & 0.002 & 2.34 \\
\hline \multirow[t]{5}{*}{ PreCG_L } & 1 & $0.002 *$ & 0.001 & 4.06 \\
\hline & 2 & $0.002 *$ & 0.001 & 2.24 \\
\hline & 3 & $0.006^{*}$ & 0.001 & 4.21 \\
\hline & 4 & 0.006 & 0.005 & 1.28 \\
\hline & 5 & $0.003 *$ & 0.001 & 3.25 \\
\hline \multirow[t]{5}{*}{ SMA } & 1 & 0.003 & 0.002 & 1.58 \\
\hline & 2 & $0.003 *$ & 0.001 & 3.30 \\
\hline & 3 & 0.003 & 0.002 & 1.98 \\
\hline & 4 & $0.007 *$ & 0.002 & 3.05 \\
\hline & 5 & $0.001 *$ & 0.001 & 2.39 \\
\hline
\end{tabular}


TABLE 6.

The variance estimates of the Level-2 random path coefficient estimates and their standard errors.

\begin{tabular}{|c|c|c|c|c|c|}
\hline \multicolumn{3}{|c|}{ The variance of $\theta_{l j}{ }^{\prime} s$} & \multirow{2}{*}{$\begin{array}{l}\text { Estimate } \\
0.009^{*}\end{array}$} & \multirow{2}{*}{$\begin{array}{c}\text { S.E. } \\
0.004\end{array}$} & \multirow{2}{*}{$\begin{array}{l}\text { C.R } \\
2.15\end{array}$} \\
\hline IPL_R & $\leftarrow$ & IPL_R & & & \\
\hline & & PreCG_R & $0.029 *$ & 0.013 & 2.27 \\
\hline & & CL_R & $0.105^{*}$ & 0.030 & 3.55 \\
\hline & & CL_L & $0.082^{*}$ & 0.034 & 2.38 \\
\hline & & IPL_L & $0.043^{*}$ & 0.017 & 2.56 \\
\hline & & PreCG_L & $0.043^{*}$ & 0.011 & 3.72 \\
\hline & & SMA & $0.030 *$ & 0.011 & 2.79 \\
\hline \multirow[t]{7}{*}{ PreCG_R } & $\leftarrow$ & IPL_R & $0.025^{*}$ & 0.009 & 2.97 \\
\hline & & PreCG_R & $0.006^{*}$ & 0.003 & 2.26 \\
\hline & & $\mathrm{CL} \_\mathrm{R}$ & $0.028 *$ & 0.008 & 3.69 \\
\hline & & CL_L & $0.075^{*}$ & 0.030 & 2.51 \\
\hline & & IPL_L & $0.028 *$ & 0.007 & 3.76 \\
\hline & & PreCG_L & $0.058 *$ & 0.017 & 3.45 \\
\hline & & SMA & $0.047 *$ & 0.016 & 2.98 \\
\hline \multirow[t]{7}{*}{ CL_R } & $\leftarrow$ & IPL_R & $0.150^{*}$ & 0.050 & 2.98 \\
\hline & & PreCG_R & $0.100^{*}$ & 0.045 & 2.21 \\
\hline & & CL_R & $0.013^{*}$ & 0.003 & 4.00 \\
\hline & & CL_L & $0.049 *$ & 0.019 & 2.59 \\
\hline & & IPL_L & $0.050^{*}$ & 0.015 & 3.27 \\
\hline & & PreCG_L & $0.030^{*}$ & 0.010 & 2.95 \\
\hline & & SMA & $0.073^{*}$ & 0.025 & 2.95 \\
\hline \multirow[t]{7}{*}{ CL_L } & $\leftarrow$ & IPL_R & $0.044^{*}$ & 0.014 & 3.07 \\
\hline & & PreCG_R & 0.096 & 0.077 & 1.25 \\
\hline & & CL_R & $0.034 *$ & 0.013 & 2.61 \\
\hline & & CL_L & $0.009 *$ & 0.003 & 3.49 \\
\hline & & IPL_L & $0.035^{*}$ & 0.014 & 2.52 \\
\hline & & PreCG_L & $0.020 *$ & 0.006 & 3.55 \\
\hline & & SMA & $0.052 *$ & 0.024 & 2.13 \\
\hline \multirow[t]{7}{*}{ IPL_L } & $\leftarrow$ & IPL_R & $0.091^{*}$ & 0.033 & 2.78 \\
\hline & & PreCG_R & $0.089 *$ & 0.024 & 3.80 \\
\hline & & CL_R & $0.072 *$ & 0.026 & 2.80 \\
\hline & & CL_L & $0.052 *$ & 0.016 & 3.17 \\
\hline & & IPL_L & $0.012^{*}$ & 0.003 & 3.76 \\
\hline & & PreCG_L & $0.053^{*}$ & 0.017 & 3.19 \\
\hline & & SMA & $0.129 *$ & 0.038 & 3.40 \\
\hline \multirow[t]{7}{*}{ PreCG_L } & $\leftarrow$ & IPL_R & $0.154 *$ & 0.046 & 3.33 \\
\hline & & PreCG_R & $0.304 *$ & 0.095 & 3.20 \\
\hline & & CL_R & $0.066^{*}$ & 0.025 & 2.68 \\
\hline & & CL_L & 0.097 & 0.051 & 1.92 \\
\hline & & IPL_L & $0.053^{*}$ & 0.013 & 3.93 \\
\hline & & PreCG_L & 0.012 & 0.006 & 1.89 \\
\hline & & SMA & $0.156^{*}$ & 0.058 & 2.71 \\
\hline \multirow[t]{7}{*}{ SMA } & $\leftarrow$ & IPL_R & 0.025 & 0.013 & 1.95 \\
\hline & & PreCG_R & $0.080^{*}$ & 0.023 & 3.48 \\
\hline & & CL_R & $0.081^{*}$ & 0.030 & 2.66 \\
\hline & & CL_L & $0.068^{*}$ & 0.029 & 2.34 \\
\hline & & IPL_L & $0.081 *$ & 0.022 & 3.60 \\
\hline & & PreCG_L & $0.054 *$ & 0.016 & 3.40 \\
\hline & & SMA & $0.008^{*}$ & 0.002 & 3.87 \\
\hline
\end{tabular}

